\title{
Studienfinanzierung - Teil 2: Darlehensangebote
}

\author{
Studienfinanzierung in Zeiten der COVID-19-Pandemie
}

\author{
Dieser Beitrag setzt den ersten Teil des Überblicks über die Studienfinanzie- \\ rung in Deutschland fort (siehe WiSt 9/2021). Wurden im ersten Teil vor \\ allem Stipendienangebote und BAföG behandelt, so werden nachfolgend \\ Darlehensangebote vorgestellt. Hierzu zählen die Hilfe zum Studienab- \\ schluss, der Bildungskredit sowie Studienkredite, die am Beispiel des \\ Studienkredits der Kreditanstalt für Wiederaufbau (KfW) erläutert werden. \\ Die Ausführungen erheben keinen Anspruch auf Vollständigkeit und \\ können eine individuelle Beratung nicht ersetzen. Auf steuerrechtliche \\ Folgen einzelner Finanzierungsformen wird nicht eingegangen.
}

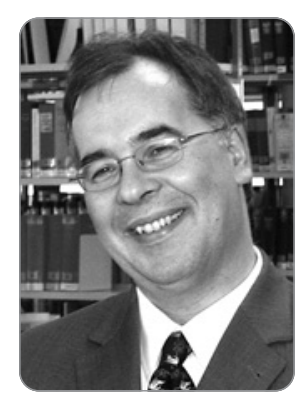

\section{Prof. Dr. Matthias Kropp}

ist Professor für Allgemeine Betriebswirtschaftslehre, insbesondere Finanzwirtschaft, an der Hochschule Pforzheim. Bevorzugte Forschungsgebiete: Internationale Rechnungslegung, Finanzmanagement und Bankbetriebslehre, insb. Bankrechnungswesen und Bankenregulierung.

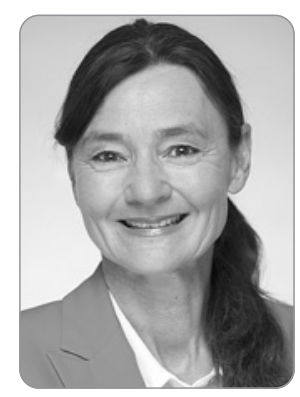

Prof. Dr. Katja Rade

ist Rektorin der Hochschule für Technik in Stuttgart. Bevorzugte Forschungsgebiete: Rechnungswesen und Controlling.

1. Die Hilfe zum Studienabschluss als zinsloses BAföGStaatsdarlehen

Das Bundesausbildungsförderungsgesetz beinhaltete in $\S 17$ Abs. 3 BAföG auch die Förderung in Form eines verzinslichen BAföG-Bankdarlehens der KfW. Diese Förderung konnten Studierende bei einer Überschreitung der Förderhöchstdauer beantragen. Das BAföG-Bankdarlehen ist seit der BAföG-Reform 2019 in der bisherigen Form entfallen und wurde stattdessen auf Basis $\S 15$ Abs. 3a i.V.m. § 17 Abs. 3 BAföG durch ein separates, zinsloses BAföG-Staatsdarlehen (Hilfe zum Studienabschluss) ersetzt.

Die Hilfe zum Studienabschluss kann bei einer Überschreitung der Regelstudienzeit um maximal vier Semester für maximal 12 Monate gewährt werden kann. Die Beantragung erfolgt im Rahmen eines BAföG-Antrags. Voraussetzung hierfür ist eine Bescheinigung der Hochschule, dass das Studium innerhalb des Förderzeitraums abgeschlossen werden kann.

Für dieses Volldarlehen gilt die Deckelung der BAföG-Schulden auf $10.010 €$ jedoch nicht, eine Studienabschlusshilfe erhöht den Rückzahlungsbetrag entsprechend. Das BAföGStaatsdarlehen als Studienabschlusshilfe ist nach einer Karenzzeit von bereits 18 Monaten (bzw. 3 Jahren, wenn zuvor kein $B A f \ddot{o} G$ in Anspruch genommen wurde) in monatlichen Raten von mindestens $130 €$ zurückzuzahlen. Wie beim normalen $B A f o ̈ G$ ist die Rückzahlung einkommensabhängig ausgestaltet und umfasst auch einen Schulderlass nach 20 Jahren.

\section{Weitere Darlehensangebote}

\subsection{Vorüberlegungen}

Studienkreditangebote zeichnen sich i.d.R. durch den Verzicht auf sonst gängige bankübliche Sicherheiten aus. 
Vor einer Kreditaufnahme sollten jedoch einige Grundüberlegungen angestellt werden (vgl. schon Kropp/Rade, 2008, S. 53): Zunächst sollte man sich darüber klarwerden, ob überhaupt und in welchem Umfang die Notwendigkeit besteht, auf einen Kredit zurückzugreifen. Die späteren Zinsund Tilgungszahlungen können das dann verfügbare Einkommen drastisch schmälern und bei hohen Kreditbeträgen im Extremfall den Weg in die Schuldenfalle vorzeichnen.

Man sollte daher zunächst einmal eine realistische Planung der monatlichen Einnahmen und Ausgaben vornehmen. Eine Checklisten hierfür liefert etwa der Studienkredittest des CHE (vgl. Müller, 2020, S. 94 f.). Dabei ist zu überlegen, ob sich nicht Einsparmöglichkeiten bei den Ausgaben ergeben: Studienkredite sollten die Investition ins eigene Studium ermöglichen, nicht aber (zusätzliche) Konsumwünsche. Führt eine zur Sicherung des Lebensunterhalts notwendige Erwerbstätigkeit hingegen $\mathrm{zu}$ einer Verlängerung der Studienzeit, so kann eine Kreditaufnahme sinnvoll sein: Eine Studienzeitverlängerung durch „Jobben“ ist häufig wesentlich teurer, gehen doch bei entsprechender Arbeitsmarktperspektive die Vollzeiteinkünfte eines Akademikers verloren.

Der Studierende sollte sich dann vorab über die Kreditangebote verschiedener Anbieter informieren und diese detailliert miteinander vergleichen. Möglichkeiten hierzu bietet der Studienkredittest des CHE (vgl. Müller, 2020), der einen umfassenden Vergleich verschiedener, auch regionaler oder auf bestimmte Studiengänge spezialisierter Anbieter enthält und auch auf alternative Bildungsfonds-Konzepte (nicht darlehensbasierte Fondsförderungen mit einkommensabhängiger Rückzahlung) eingeht.

\subsection{Der Bildungskredit}

Für Studierende, die nicht $B A f o ̈ G$-antragsberechtigt sind oder deren BAföG-Förderung eher gering ist, kann der Bildungskredit eine Alternative bieten. Der Bildungskredit wird durch die KfW nach Bewilligung durch das Bundesverwaltungsamt (vgl. Bundesverwaltungsamt) gewährt. Antragsberechtigt sind Studierende mit deutscher oder EU-Staatsbürgerschaft sowie deren Angehörige, die an staatlichen bzw. staatlich anerkannten Hochschulen ein Vollzeitstudium absolvieren und deren Höchstalter bei Antragstellung 36 Jahre nicht überschreitet. Antragsberechtigt sind zudem Studierende von Master-, Aufbau-, Zusatz- und Ergänzungsstudiengängen. Je nach Studium bestehen weitere Voraussetzungen, so müssen Bachelorstudierende eine evtl. bestehende Vorprüfung bestanden bzw. die Leistungen des ersten Studienjahres vollständig erbracht haben und nicht länger als 12 Semester studieren (oder aber zur Abschlussprüfung zugelassen sein).
Bei Antragstellung sind neben der Immatrikulationsbescheinigung einige weitere Nachweise zu erbringen, weitere Leistungsnachweise nach der Bewilligung des Darlehens sind nicht erforderlich. Da das Darlehen ohne bankübliche Sicherheiten (z.B. eine Bürgschaft der Eltern) vergeben wird, führen eindeutig negative Bonitätsmerkmale, wie z.B. ein Insolvenzverfahren zu einer Ablehnung des Antrags.

Der Bildungskredit kann in monatlichen Raten von $100 €$, $200 €$ und - maximal - $300 €$ pro Monat über höchstens 2 Jahre ausgezahlt werden. Hieraus ergibt sich ein maximal möglicher Kreditbetrag von 7.200 $€$, mindestens muss ein Kredit von $1.000 €$ in Anspruch genommen werden. Höchstens die Hälfte des Kreditbetrags kann bei Bedarf in einer Summe als Abschlagszahlung beantragt werden.

Der Bildungskredit kann mit BAföG oder dem KfW-Studienkredit kombiniert werden. Dies ist auch erforderlich, da er aufgrund seiner begrenzten Höhe regelmäßig nicht allein zur Deckung der Lebenshaltungskosten ausreichen wird. Da Bachelor-Studierende nach erfolgreichem Abschluss aller Studienleistungen des ersten Studienjahres auch nach Überschreiten der BAföG-Förderdauer das Darlehen erhalten können, eignet sich der Bildungskredit als Studienabschlusskredit.

Auf die während der Auszahlungsphase ansteigenden Kreditbeträge werden variable Zinsen berechnet, die an die Entwicklung des 6-Monats-EURIBOR (Stichtage 1. April und 1. Oktober) mit einem Zuschlag von $1 \%$-Punkt p.a. gebunden sind. Am 1.10.2020 betrug der Effektivitätszins 0,52 \%. Der Kredit ist in den ersten vier Jahren seiner Laufzeit zinsund tilgungsfrei (Karenzphase) und anschließend einschließlich der aufgelaufenen Zinsen während der Rückzahlungsphase in gleichbleibenden Raten von $120 €$ pro Monat einkommensunabhängig zurückzuzahlen. Eine vorzeitige Rückzahlung - ganz oder teilweise - ist jederzeit ohne zusätzliche Kosten möglich. Der Bildungskredit ist damit vergleichsweise günstig, unterliegt aufgrund der halbjährlichen Zinsanpassung jedoch einem Zinsänderungsrisiko.

Die aktuelle Pandemie hat nicht zu einer Änderung der Konditionen des Bildungskredits geführt. Bei Nachweis einer finanziellen Notlage kann wie bisher eine Stundung von Zins- und Tilgungszahlungen bzw. die Reduzierung der Rückzahlungsraten beim Bundesverwaltungsamt beantragt werden.

Neben dem Bildungskredit bieten auch andere i.d.R. regionale Anbieter Studienkredite zum Studienabschluss an (vgl. Müller, 2020, Kapitel 6).

\subsection{Der KfW-Studienkredit}

Die nachfolgende Darstellung konzentriert sich auf den KfW-Studienkredit (vgl. KfW, 2021), da gemäß CHE-Studienkredittest ein Großteil aller Studienkreditfinanzierungen 
auf diesen Kredit entfallen. Eine Kombination mit dem Bildungskredit oder BAföG ist möglich. Bankübliche Sicherheiten sind beim KfW-Studienkredit nicht erforderlich. Der Kredit kann online beantragt werden, wird aber über Vertriebspartner (Banken und Sparkassen, Studentenwerke) abgewickelt, die u.a. die Prüfung der einzureichenden Unterlagen übernehmen.

Der KfW-Studienkredit richtet sich an Studierende staatlicher bzw. staatlich anerkannter deutscher Hochschulen, gefördert werden nicht nur Vollzeit- sondern auch Teilzeitstudien und berufsbegleitende Studien. Die geförderten Studiengänge sind nicht auf Bachelorstudiengänge begrenzt, sondern umfassen auch Masterstudiengänge sowie andere postgraduale Studien und auch ein Promotionsstudium.

$\mathrm{Zu}$ den persönlichen Voraussetzungen für die Kreditgewährung zählen neben der Volljährigkeit und einer Studienbescheinigung zunächst die deutsche Staatsangehörigkeit, wobei auch Angehörige anderer EU-Staaten einen Studienkredit beantragen können, sofern sie seit mindestens drei Jahren in Deutschland leben. Dies gilt mit Einschränkungen auch für Familienangehörige dieser beiden Gruppen unabhängig von ihrer eigenen Nationalität. Antragsberechtigt sind ebenso sog. Bildungsinländer mit inländischer Meldeadresse, die eine deutsche Hochschulzugangsberechtigung im Inland oder an einer deutschen Schule im Ausland erworben haben. Antragsberechtigt waren bis zum 15.02.2021 im Rahmen der Übergangshilfe bei pandemiebedingter Notlage alle ausländischen Studierenden unabhängig von ihrer bisherigen Aufenthaltsdauer in Deutschland; ob diese Ausnahmeregelung verlängert wird, war bei Erstellung dieses Beitrags noch nicht abzusehen. Ein Antragsteller muss spätestens im Höchstalter von 44 Jahren mit seinem Studium begonnen haben und maximal im 10. Fachsemester studieren.

Eindeutig negative Bonitätsmerkmale führen zu einer $\mathrm{Ab}$ lehnung des Antrags. Ein Studiengangwechsel zählt nicht als negatives Bonitätsmerkmal, verkürzt aber die mögliche Auszahlungsphase.

In der Auszahlungsphase beträgt die monatliche Mindestinanspruchnahme $100 €$ und der monatliche Höchstbetrag $650 €$, wobei der Studierende innerhalb dieser Grenzen halbjährlich variieren kann. Es ist jedoch zu beachten, dass eine Zinsverrechnung stattfindet, so dass die tatsächliche Auszahlung bei konstanter Inanspruchnahme im Zeitverlauf permanent sinkt. In späteren Studienphasen kann mit der $K f W$ ein Zinsaufschub vereinbart werden, so dass eine solche Zinsverrechnung entfällt. Auf die während der Auszahlungsphase ansteigenden Kreditbeträge werden variable Zinsen berechnet, die an die Entwicklung des 6Monats-EURIBOR (Stichtage 1. April und 1. Oktober) gebunden sind und auf diesen einen festen Zuschlag vorse- hen. Der Student unterliegt damit auch hier einem Zinsänderungsrisiko, das sich bei der üblichen Zinsverrechnung in nur bedingt voraussehbaren tatsächlichen Monatsauszahlungen niederschlägt. Als Übergangshilfe bei pandemiebedingter Notlage hat die Bundesregierung alle Zinsen während der Auszahlungsphase bis zum 31.12.2021 übernommen. Es kommt so bis Ende 2021 nicht $\mathrm{zu}$ einer weiteren Kürzung der Auszahlungsbeträge durch anwachsende verrechnete Zinsen bzw. nicht zu einem entsprechenden Anwachsen des Kreditbetrags bei einer Stundung.

Die Länge der Auszahlungsphase ist vom Alter bei Finanzierungsbeginn abhängig und beträgt bei einem Alter von 24 Jahren zunächst fünf Jahre und kann um weitere zwei Jahre bei Vorlage eines Nachweises der Hochschule über den voraussichtlich erfolgreichen Studienabschluss verlängert werden. Mit zunehmendem Lebensalter bei Studienbeginn fällt die Auszahlungsphase auf maximal 3 Jahre (bei einem Alter von 44 Jahren). Bei voller Ausnutzung des monatlichen Höchstbetrags von $650 €$ über den maximalen Auszahlungszeitraum von sieben Jahren beläuft sich der Höchstkreditbetrag auf $54.600 €$ zuzüglich evtl. gestundeter Zinsen.

Zu den Kontrollen während der Auszahlungsphase zählt die laufende Einreichung von Studienbescheinigungen zum Nachweis der Immatrikulation. Bei Bachelor-Studierenden wird spätestens nach Ablauf des 6. Fördersemesters einmalig ein Leistungsnachweis gefordert, aus dem bei einem Vollzeitstudium hervorgeht, dass mindestens die Hälfte der für den Studienabschluss erforderlichen ECTSCredits erfolgreich erbracht wurde. Negativmerkmale wie Exmatrikulation, Nichteinreichung von Studienbescheinigungen oder geforderter Leistungsnachweises führen ebenso wie negative Bonitätsmerkmale zu einem Stopp der Auszahlungen und zum Übergang in die Rückzahlungsphase bzw. im Extremfall zur Kreditkündigung.

Der KfW-Studienkredit sieht im Anschluss an die Auszahlungsphase eine Karenzzeit von sechs bis 18 Monaten (bzw. 23 Monaten bei Studienabschluss innerhalb eines Semesters) nach Wahl des Kreditnehmers vor. Während dieser Karenzzeit wird der Kredit weiter variabel verzinst und es sind nur Zinszahlungen zu leisten. Die Zeit der Arbeitssuche im Anschluss an ein Studium oder die Zeit eines Referendariats können so rückzahlungsfrei überbrückt werden. Der KfW-Studienkredit verzinst sich auch während der Rückzahlungsphase weiterhin variabel. Zu jedem Zinsanpassungstermin hat der Studierende jedoch unter bestimmten Voraussetzungen das Recht, einen Festzins für die Restlaufzeit des Darlehens, maximal jedoch 10 Jahre, zu vereinbaren (Festzinsoption). Studierende können so ein Zinsänderungsrisiko in der Rückzahlungsphase ausschließen und ihre Zahlungsverpflichtungen für den Zeitraum der Zinsfestschreibung fest planbar gestalten. 
Die Rückzahlung erfolgt in Form einer Annuität, d.h. es werden gleichbleibende Raten vereinbart, wobei sich der Zinsanteil bei konstanter Verzinsung zu Gunsten des Tilgungsanteils fortlaufend vermindert. Die Höhe der Annuität beträgt mindestens $20 €$ im Monat. Eine höhere Monatsrate ist dann erforderlich, wenn das Darlehen ansonsten nicht innerhalb der maximalen Rückzahlungsdauer von 25 Jahren bzw. bis zum 67. Lebensjahr zurückgezahlt werden könnte. Die Annuität kann bei variabler Verzinsung im Verlauf der Rückzahlungsphase erhöht und auch wieder auf die Mindesthöhe abgesenkt werden. Die laufende Belastung kann z.B. zunächst geringer gewählt werden, um finanzielle Spielräume z.B. zur Tilgung eines parallel in Anspruch genommenen BAföG-Darlehens oder Bildungskredits $\mathrm{zu}$ erhalten. Sondertilgungen sind halbjährlich $\mathrm{zu}$ jedem Zinsanpassungstermin ohne zusätzliche Kosten möglich auch bei einer Festzinsvereinbarung. Dies ermöglicht es dem Kreditnehmer, Einmalzahlungen des Arbeitgebers (Tantieme, Boni) zur beschleunigten Tilgung des Studienkredits zu verwenden.

\section{Fazit}

Die Finanzierung durch die Eltern und die eigene Erwerbstätigkeit stellen für die meisten Studierenden die wichtigsten Einkommensquelle dar. Eine eigene Erwerbstätigkeit ("Jobben“) mit studienbezogenem Charakter kann zu einer sinnvollen berufsbezogenen Qualifizierung führen. Kritisch ist jedoch ein Umfang an Erwerbstätigkeit zu sehen, der zu einer Studienzeitverlängerung führt, oder gar den erfolgreichen Studienabschluss gefährdet. Kann oder soll der Lebensunterhalt nicht aus den genannten Quellen bestritten werden, etwa aufgrund einer pandemiebedingten finanziellen Notlage, so kommt dem BAföG eine bedeutende Rolle zu. Kritisch ist hier jedoch häufig die Förderdauer, wenn sich aufgrund des Auslaufens des BAföG eine massive Finanzierungslücke ergibt.

Zur Überbrückung finanzieller Engpässe in späteren Studienphasen können das BAföG-Staatsdarlehen oder der
Bildungskredit eine alternative Finanzierungsquelle sein. In anderen Fällen mag erst ein Studienkredit ein Studium ermöglichen. Die Inanspruchnahme von Darlehen und Krediten verlangt jedoch auch Disziplin: Ein allzu laxer Umgang kann sich schnell in hohen Kreditbeträgen am Ende des Studiums niederschlagen und den Übergang ins Erwerbsleben schwer belasten, ja sogar in die Schuldenfalle führen.

$\mathrm{Zu}$ Unrecht eher wenig bekannt scheinen die Finanzierungsmöglichkeiten über Stipendien. Schon allein aufgrund des finanziellen Vorteils einer fehlenden Rückzahlungsverpflichtung lohnt sich die Beschäftigung mit den vielfältigen bestehenden Möglichkeiten, die sich nicht nur an „Überflieger" richten.

\section{Literatur}

Bundesgesetz über individuelle Förderung der Ausbildung (Bundesausbildungsförderungsgesetz - BAföG), in der Fassung der Bekanntmachung vom 7. Dezember 2010 (BGBl. IS. 1952; 2012 I S. 197), zuletzt durch Artikel 4 des Gesetzes vom 12. November 2020 (BGBl. I S. 2416) geändert, Online, URL: https://www.gesetze-im-internet.de/baf_g/ (Abrufdatum: 30.12.2020).

Bundesministerium für Bildung und Forschung (BMBF, 2021), Online, URL: https://www.bafög.de/ (Abrufdatum: 31.01.2021).

Bundesverwaltungsamt, Online im Internet: URL: https://www.bva.bund. de/DE/Services/Buerger/Schule-Ausbildung-Studium/Bildungskredit/bi ldungskredit_node.html (Abrufdatum: 05.02.2021).

Kreditanstalt für Wiederaufbau (KfW, 2021), Online, URL: https://www.kf w.de/inlandsfoerderung/Privatpersonen/Studieren-Qualifizieren/Finanz ierungsangebote/KfW-Studienkredit-(174)/ (Abrufdatum: 06.02.2021).

Kreditanstalt für Wiederaufbau (KfW, 2021), Merkblatt Studium und Beruf - KfW-Studienkredit, Online, URL: https://www.kfw.de/PDF/Download-C enter/F\%C3\%B6rderprogramme-(Inlandsf\%C3\%B6rderung)/PDF-Dokume nte/6000002590_M_174_Studienkredit.pdf (Abrufdatum: 06.02.2021). Kropp, Matthias/Rade, Katja, Studienkredite zur Finanzierung des Lebensunterhalts, in: WiSt - Wirtschaftswissenschaftliches Studium, 37. Jg. (2008), S. 52-55.

Müller, U., CHE-Studienkredit-Test 2020 - Studienkredite, Abschlussdarlehen und Bildungsfonds im Vergleich, CHE Centrum für Hochschulentwicklung $g \mathrm{GmbH}$, Arbeitspapier Nr. 229, Juli 2020, Gütersloh, Online, URL: https://www.che.de/download/che-studienkredit-test-2020/(Abrufdatum: 05.02.2021). 